\title{
Enhanced VOT on Foggy Videos Using Probabilistic Estimation Scheme
}

\author{
Harjeet Kaur and Silky Narang \\ Department of Computer Science and Engineering Department, Chandigarh University, \\ National Highway 95, Mohali - 140413, Punjab, India; \\ harjeetkaur2890@gmail.com, silkynarang94@yahoo.com
}

\begin{abstract}
Objectives: Visual object tracking allude the process for tracking the path of the object in the video sequence. Tracking of the object under the foggy environment is the challenging task in the video sequence. There is a great need to implement spectral analysis for the visual attention tracking model. The proposed methodology deals with object tracking using Dubbed Density of Fog Assessment based Defogger (DEFADE) filter to resist environment based detail restoration. Methods/Statistical analysis: DEFADE guided filter is used to improve the visual detail of the frame. Then frames are moved to learning network where particle region is detected and then is registered using Binary Pixel Distance Mapping (BPDM). Further Calculation of color values for the given object or selected object in the entire three channels RGB (Red, Green, Blue). The spectral analysis of visual attention tracking model is compared with the proposed approach using BPDM_CMF model. In this system an existing filter for binary conversion and color filtering was used to evaluate and estimate the trajectory of the tracked objects in foggy weather affected videos. In case of visual tracking and object detection, multi-scale detection methods constitute to combine the effort of object detection in spatial scale using BPDM with Color Mean Filter (CMF) and a defogging based spurious response inherent method for multiscale removal of fog in tracking videos and improve the fine edge tracking with reduce error probability detection between frames in a temporal tracking method. Findings: The execution of the proposed framework has been contrasted and the before technique. The proposed method works more efficiently under the foggy environment for tracking the objects in the video sequence. Application/Improvements: Automatic Recognition of the object in the video sequence. Defogging has been efficiently used in identifying the humans on the basis of trajectory.
\end{abstract}

Keywords: Color Mean Filter (CMF), DEFADE, Hue Saturation Value (HSV), Kalman Filter, Root Mean Square Error (RMSE), Tracking

\section{Introduction}

Tracking of object in videos is an important domain of research in computer field that deals with the processing of large amount of data in form of videos. Video is fundamental collection of frames that are moved at such a speed that human eye perceives that they are actually moving. The basic issue occurs when there is some occlusion while tracking some particular individual; the target may be lost in such cases proper algorithm needs to be developed ${ }^{1}$. The Singular Value Decomposition (SVD), a standout amongst the most helpful devices of direct polynomial math, is a factorization and estimation method which adequately lessens any network into a littler invertible and square lattice 2 . Various systems to provide a cost effective solution, but the rate of successful operation is bad. Inductive loop detectors installed under surface, this fails in case of poor road condition and so. In the other

${ }^{*}$ Author for correspondence 
system, the density is found by installing IR detectors along the side of the lane ${ }^{3}$.

Video order is a rising pattern that is expected to arrange the Advertisement Analog/Digital/Digital (ADD) recordings from the TV program. Grouping of ADD recordings from the general projects gives a productive way to deal with oversees and uses the ADD video information. Location of ADD video assumes a noteworthy part for notice content administration, notice for focused clients, questioning, recovering, embeddings, and avoiding the ad to see the coveted channels ${ }^{4}$. Occlusion occurs basically due to large number of persons present in the video. To handle such problems like occlusion research has been done to make the algorithms more robust to the target noise and occlusion of the target. Huge part of tracking system deals with the target color, structure, movement of the object, environment in which the object is being tracked $\underline{5}$.

There are more reasonable advanced video obtaining gadgets in the market. In view of the progress in innovation this implies more applications for computerized video. Having seen the achievement of web camera applications trust that computerized video will soon turn into a piece of regular day to day existence. Not at all like still pictures, have video arrangements given more data about how articles and situations change after some time, however at the cost of expanded space for capacity and more extensive transfer speed for transmission ${ }^{6}$.

Hence, the point of video pressure has drawn increasingly considerations amid late years. The Moving Picture Experts Group (MPEG) 4 standard proposes the utilization of protest planes in video pressure. Thus, calculations of following items are required. Additionally, with regards to surveillance there was a robot innovation ${ }^{?}$.

In computer sight tracking is an important exacting detail. It perceives the protest conduct rather than regulate Personal Computer (PC) by human machine minder. It centers to site moving articles in a video record. Bore of the picture and plan of the picture are refined by improvement of high status of the imaging sensor and utilizing object following the plague development in calculation power is a key to perform of new great calculation ${ }^{8}$.

The perfectly detecting objects can be an assert task, in view of objects can have kind of perplexing struc- tures and modifying in fiddle, size, area and adjustment over proximate video outlines. Different advancements have been gotten in the couple of decades, that can track questions in a specific video arrangement and every calculation has their own endorse and imperfections? ${ }^{9}$. The protest of intrigue which will eventually bring about a hill by protest following calculation will contain exclusion and the conspicuous techniques ought to have the capacity to minimize this hill with the end goal that the tracker is exact over the time period of the capacities. The critical complaint that needs to perceive while leading a video tracker are the point at which the foundation is rise which is like intrigued question which are moment in the stage. This episode is known as group ${ }^{10}$.

Fog is a getting of ice wrench or water hung noticeable all around at or close to the earth surface. Stratus block is as haze in the cloud. Haze is set apart from dampness just by its thickness. Clarity and diversity reduces by fog. Various methods are used to reform to improve the trait of the images and videos. In an image successive steps are applied for image processing. Basically advance the picture from apparent world and change over into framework lucid form ${ }^{11}$. Different sorts of commotion are utilized which impact the picture. In this manner picture upgrade prepare for enhancing the nature of a picture is required and subsequent to settling the nature of a picture again reestablishes that picture and video. At incite innovation for mist or cloudiness expulsion are of two sorts. The Fog rectification depends on revision of inconsistency level. Shade immersion esteem is connected on shading adjustment method and Color revision handle create transmission guide and gauges environmental light. Fog redress handle enhances the nature of foggy pixel ${ }^{12}$. In mist evacuation case the mist level over a picture is distinguish and removed.

In the enclosure of computer sight image defogging is a significant task. There are many shots in which defogging calculations are required, for example, motorized observing frameworks, programmed guided vehicle frameworks, outside question acknowledgment and visual investigation in low perceivability environments $s^{\underline{13}}$. The objective of defogging standard is to recapture the specifics of scenes from foggy pictures. 
Fog and haze are a common phenomenon on land and ocean. In foggy and hazy weather, there are many atmospheric particles of significant size. They not only absorb and scatter the reflected light of the scene, but also scatter some atmospheric light to the camera ${ }^{14}$. The image acquired by the camera is degraded and usually has low contrast and poor visibility. This will seriously influence the visual system especially the visible light visual system. Due to the degradation of the image, the targets and obstacles of the image are difficult to detect. This is terrible for mechanized video preparing, for example, highlight extraction, target following, and acknowledgment of articles. This is also one of the main reasons for accidents in the air, on the sea, and on the road. So it is important to design an image defogging algorithm to improve the environmental adaptability of the visual system ${ }^{15}$. In video data the Object discovery and following need to reduce the objective protest and way that question in progressive edges. Object tracking radically evoke supposing the area of a specific district in progressive casings in a video cycle ${ }^{16}$.

\section{Main Challenges in Video Tracking}

\subsection{Atmosphere Light}

It is comprehensible to change in power, course and shade of air light in rise of fixated protests in a video outline plane.

\subsection{Noise}

In the procuring procedure of video, it might conceivable to present a specific measure of commotion in the picture or video flag. The measure of commotion relies on sensor qualities which are utilized as a part of obtaining the video.

\subsection{Occlusions}

In moving article may fall behind some other protest which is available in the present stage. All things considered tracker may not assess the intrigued question. This is known as impediment.

\section{Applications}

- Automatic Recognition of the object.

- Identification of humans based on trajectory of the motion.

- Detection of suspicious acts at particular instant of time.

- Monitoring of real time traffic.

- Avoidance of some mis-happenings to occur.

- Recognition of gestures of the individuals.

\section{Methodology}

The proposed methodology deals with decomposing the video into image frame then preferred range of video frames or image frames from where tracking and defogging are to be done. Then frame of interest is selected i.e. the frame containing the object to be tracked. Then system is to be programmed to select the required filtered size according to size of the resolution of the video frame. Now it has been extract the mean variance of the minimum intensity pixels detected by the minimization command. These values are computed from all the three layers of the image frame. The image frames are saturated globally by applying a median symmetric filter using a window size of pre computed patch size in the first or second size. Then the inverse light restoration is applied using the symmetric median values. The restoration is inversely proportional to the compensation light. Factor is equal to one by computed light.

In order to restore we reduce the computed light and then enhance the original data by multiplication of restoration factor. For all the three layers restored in the above steps we will compute the mean value. Then proposed method, deals with finding the log of the restored and $\log$ of the original values. Then find out the mean of $\log$ restored. Calculate the standard deviation and then find out the restoration power. Highest order of derivative is selected. Then the original foggy image is restored using the highest order derivative and the processed image. Then the visibility and enhance the chrominance using the weight map are computed.

It has been pre-process the selected frame by implementing the hybrid DEFADE filter. Then find the low 
intensity pixels and create patches and apply saturation filtering to analyze image frame for separating the high detail and low detail areas. The third set is to implement the contrast on the saturation filtered image. This filter will balance the color and brightness values of the saturation enhanced image frame. In auto enhance feature will balance the enhancement done by contrast using the most bright pixel data through calculation of bright channel the image.

This will be accompanied with dilation filter based on median value extraction concept. This will reduce the sharp edge value and will be succeed by calculation of log mean values. The logs mean values will be convoluted to the original image frame with saturation values contrast enhanced values and bright pixels channel power.

\section{Results and Discussions}

Results simulated from Matrix Laboratory (MATLAB) for Visual Object tracking using pre enhancement DEFADE filtering with BPDM_CMF based probability estimation:
Results show the proposed method is more efficient in tracking the multiple objects efficiently. The time taken while processing or tracking of objects is less as compared to the earlier methods. DEFADE filter is used for defogging and BPDM_CMF is the BPDM and CMF. Implement the DEFADE guided filter to improve the visual detail of the frame. The frame is send to learning network where particle region is detected and is registered using Binary Pixel Distance Mapping. Calculate the value of colors for the given object in the entire three channels RGB and find the mean average color detail. Calculate the value of target object pixels using salient reason detection and calculate the time response of system, position of object track and AER.

DEFADE is used for defogging the videos. DEFADE achieves better results for darker dense foggy images as well as on standard foggy images. BPDM_CMF is BPDM and CMF.

Figure 1, shows the foggy video that needs to be defogged for the efficient tracking of object. The proposed technique is based on the DEFADE filter to remove the

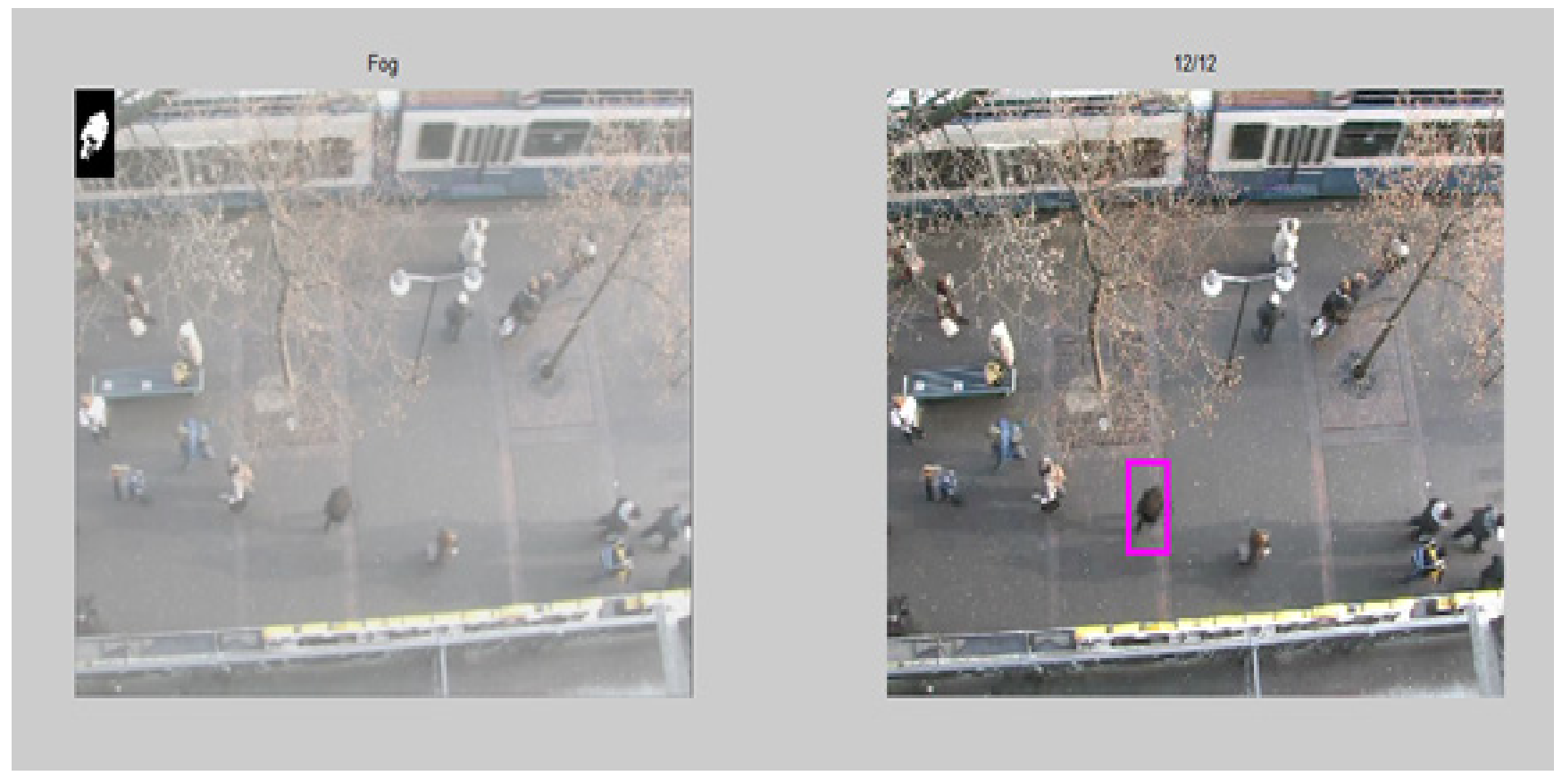

Figure 1. Selection of object in video. 
fog from the video sequence. BPDM_CMF filter is used for tracking. Thirty frames are being studied in this video sequence. Various parameters have been studied for analyzing the performance.
Figure 2, shows the time taken for tracking the appropriate gadget in the video. The average time required to track the object in the video arrangement.

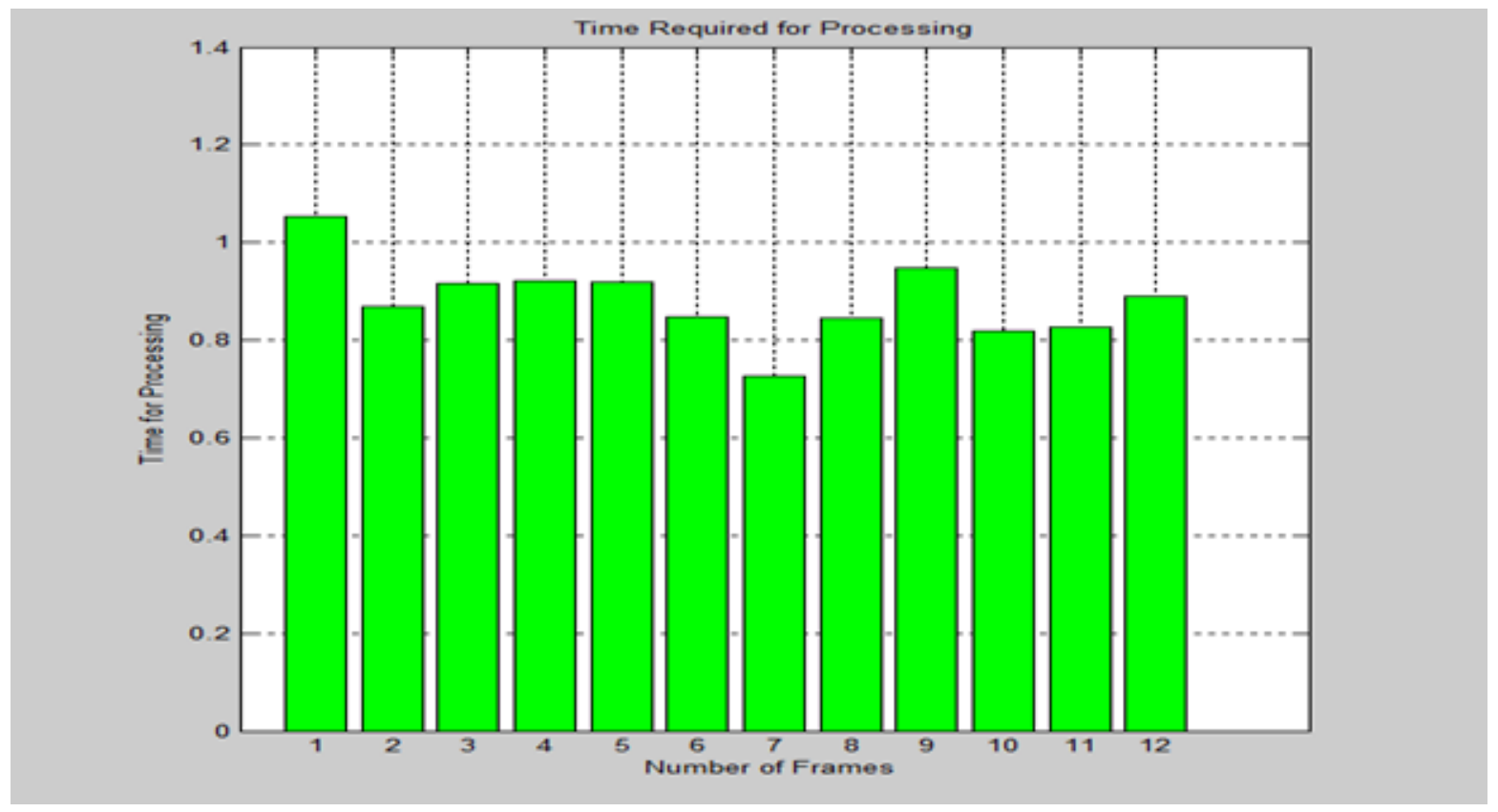

Figure 2. Time taken to track the object per frame.

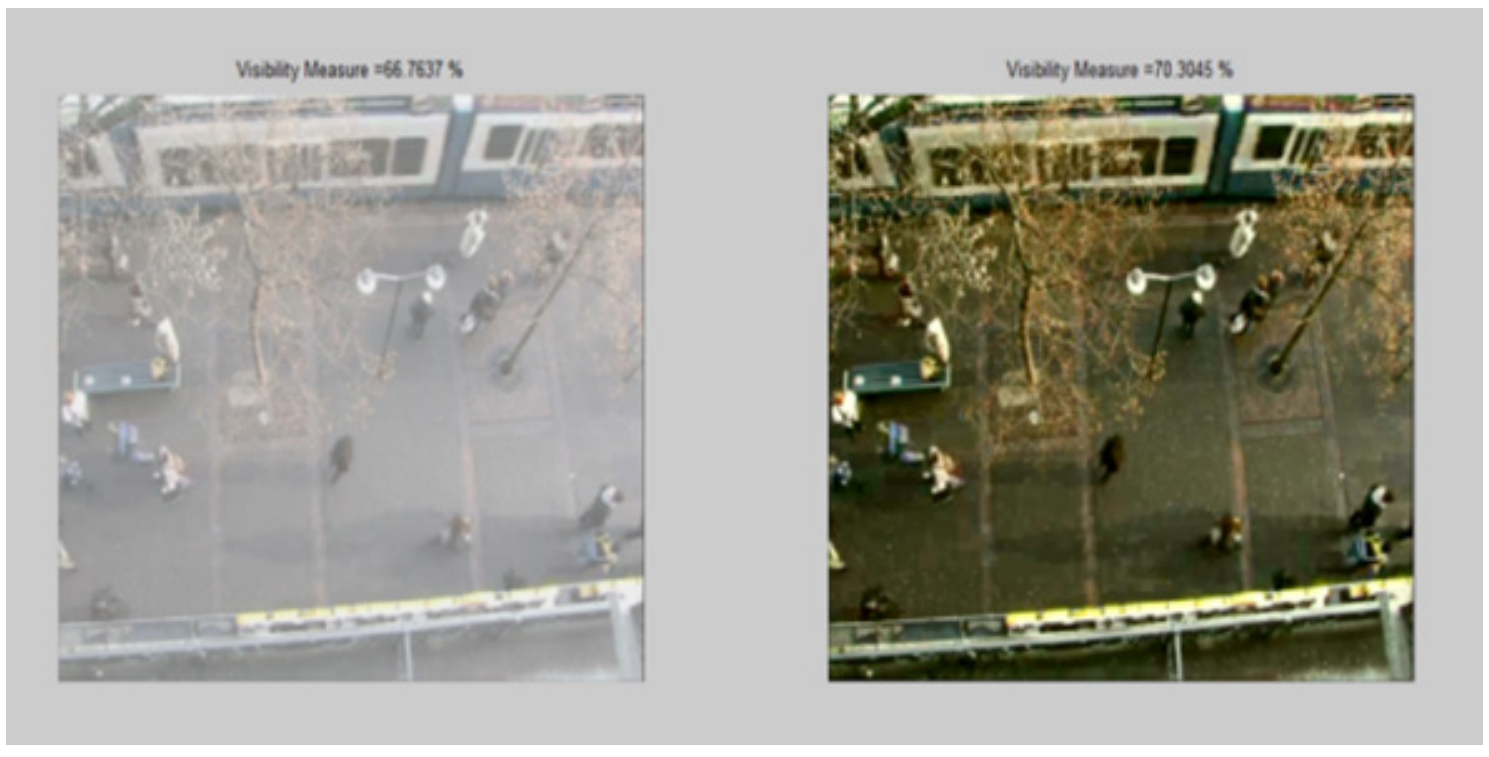

Figure 3. Visibility metric of foggy and defogged video. 
Figure 3, shows the visibility metrics for the video sequence. Visibility measure of foggy video is $66.7637 \%$ and the visibility measure for the defogged video is $70.3045 \%$. Visibility of the defogged video is more than the defogged video.
Figure 4, shows the extraction of the RGB frame. The extraction of the RGB frame into the hue, saturation and the intensity. RGB color space is represented by the RGB color.

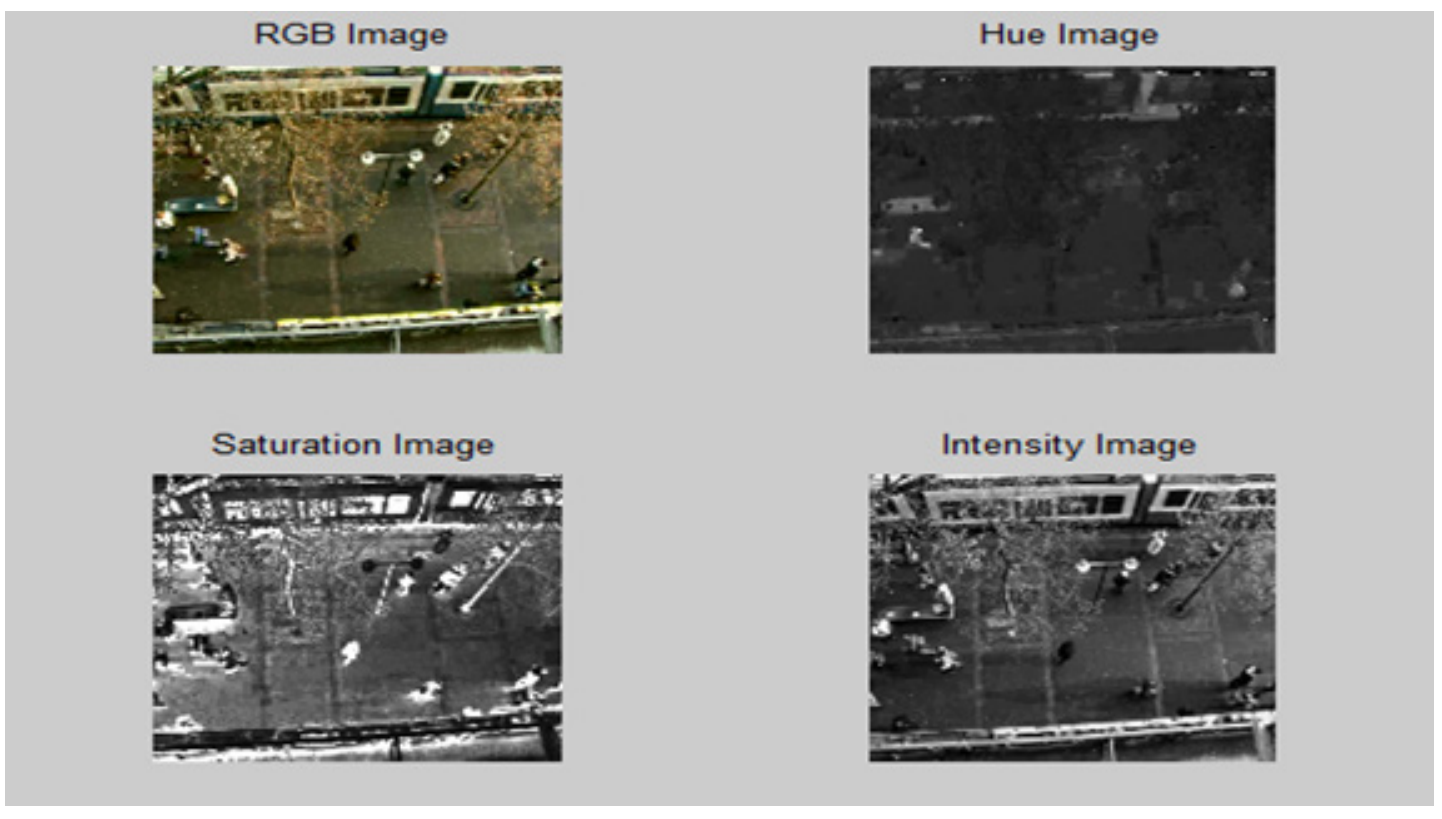

Figure 4. Extraction of RGB frames into hue, saturation and intensity.

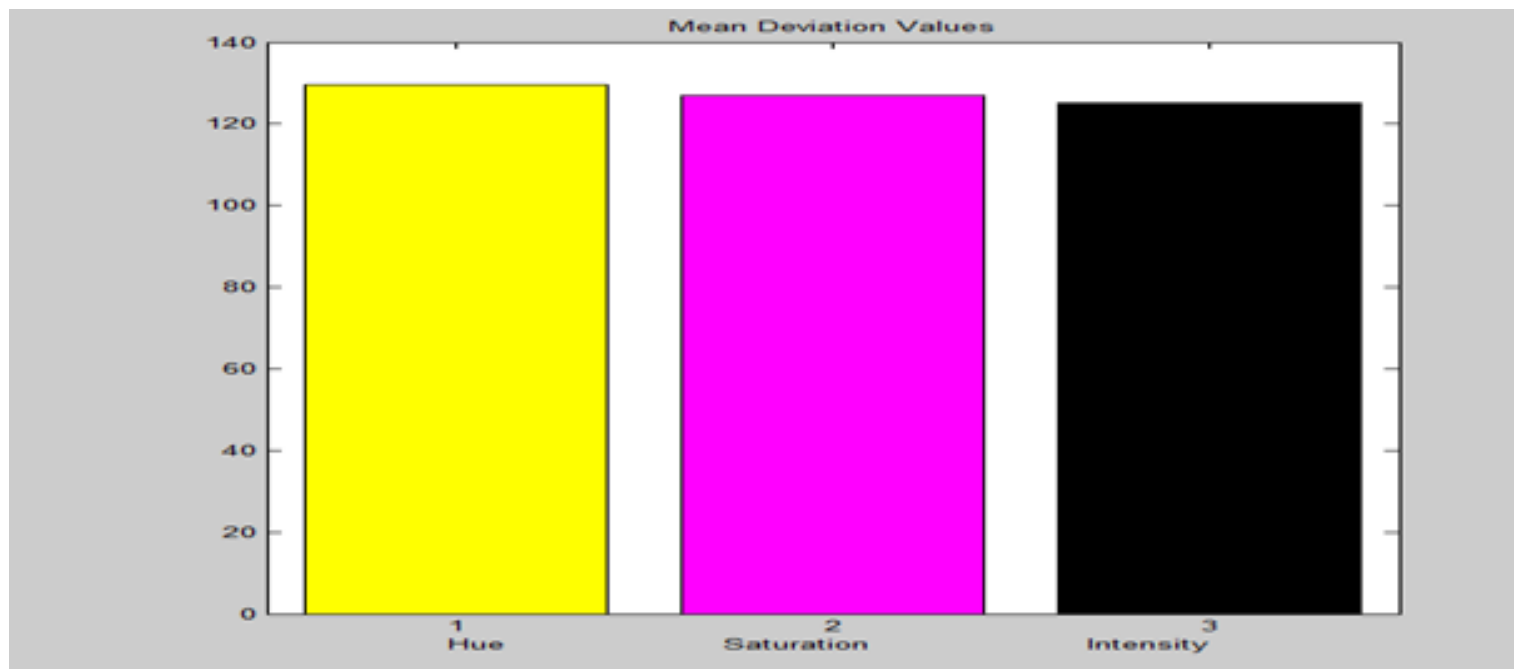

Figure 5. Mean deviation value of hue, saturation and intensity. 
Figure 5, shows the value of the mean deviation for the video sequence. Value of the hue is 125 , for saturation is 120 and the value of intensity is 99 .
Figure 6, shows the mean square error value for the proposed system.

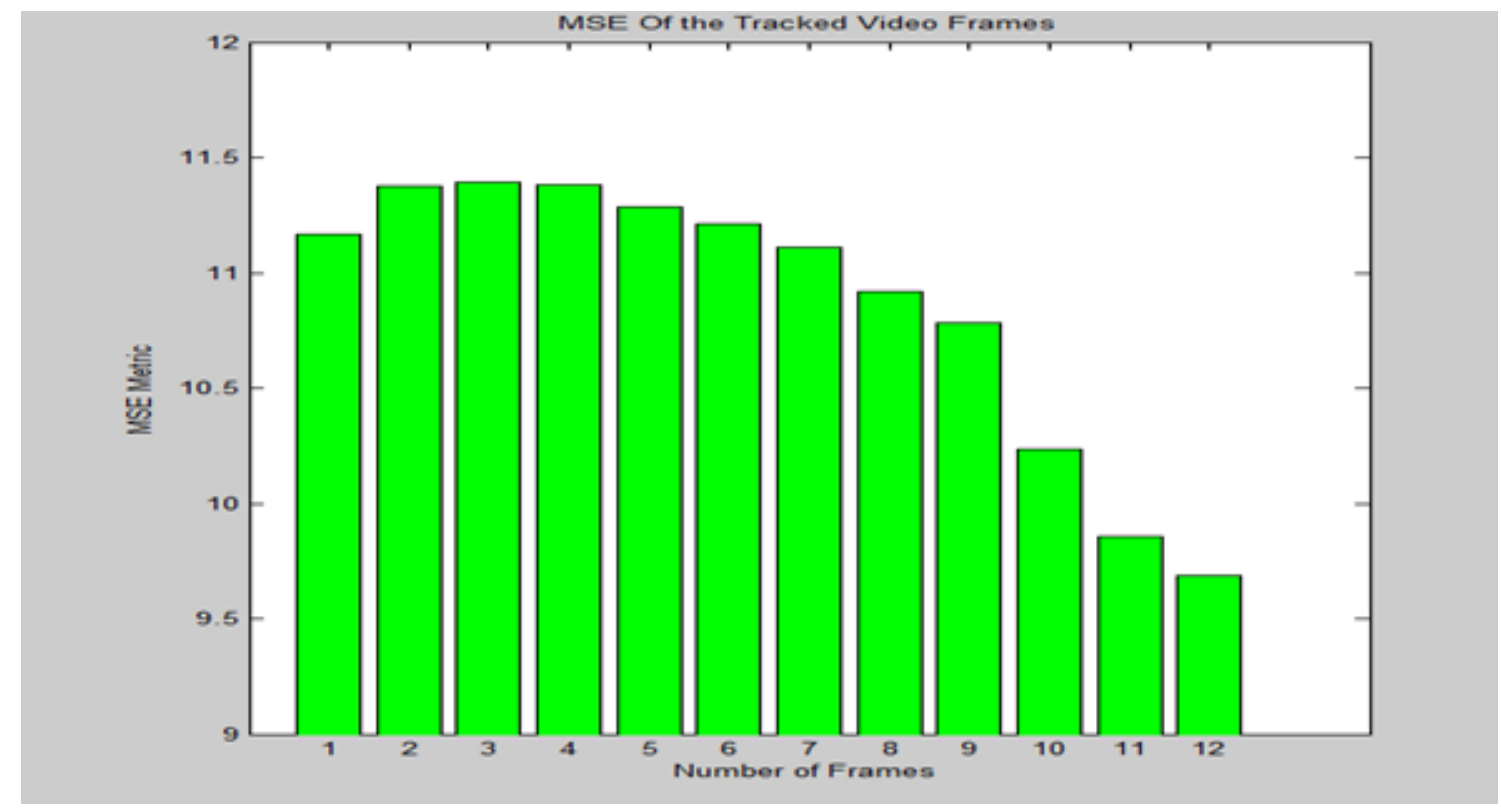

Figure 6. Mean squared error per frame.

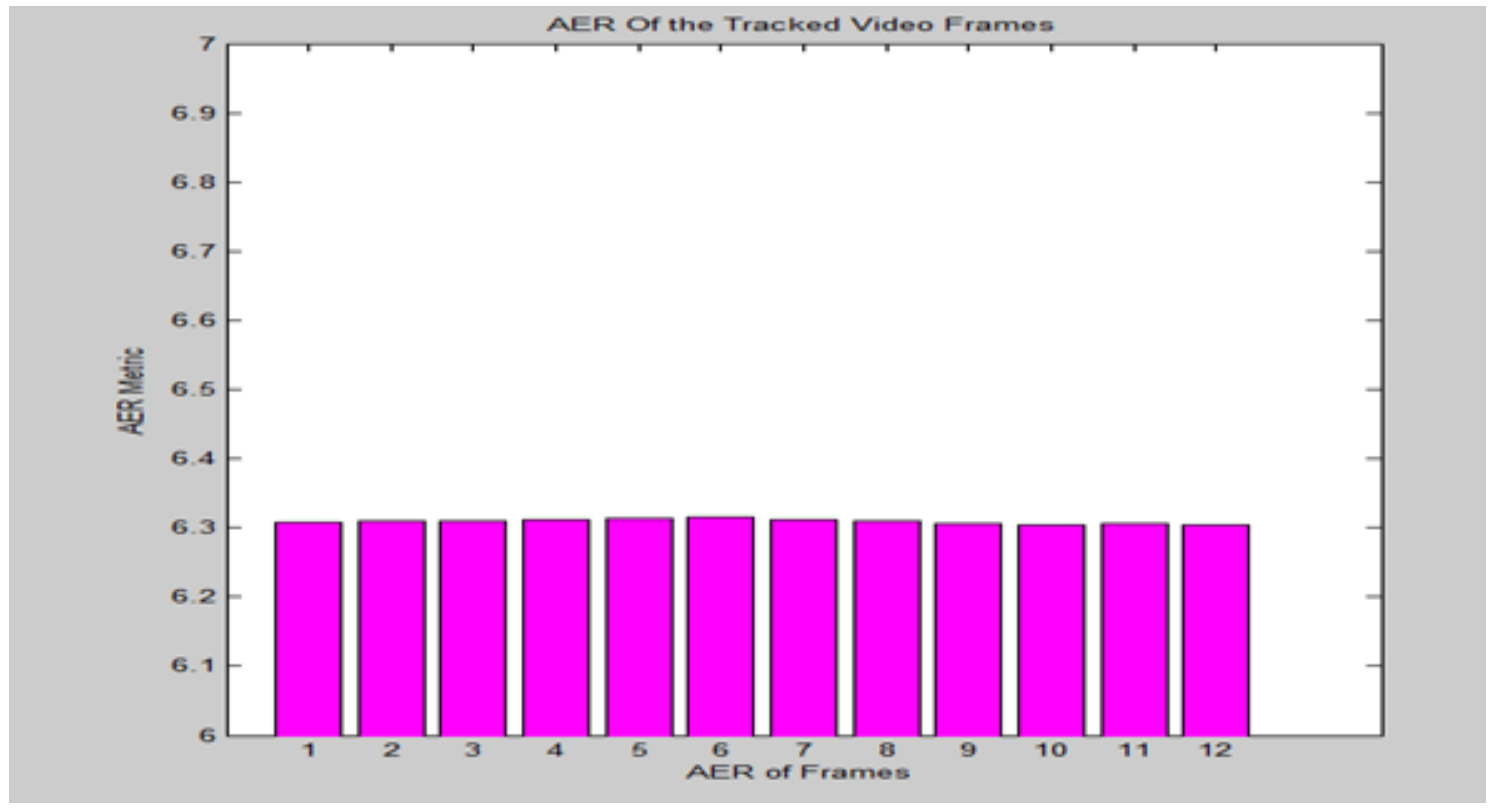

Figure 7. AER of the restored video frame. 


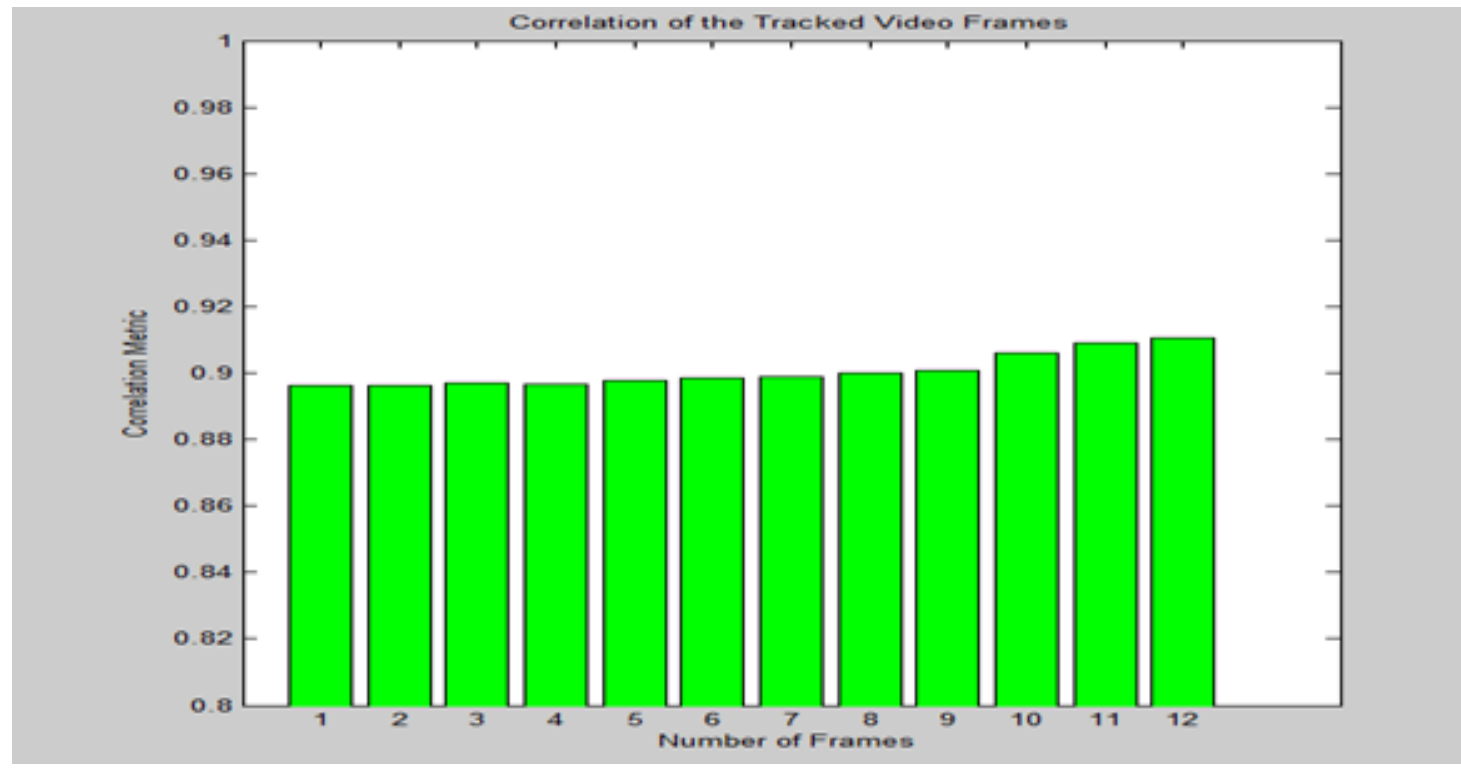

Figure 8. Correlation of the restored video frames.

Figure 7, shows the AER for the proposed system. Average error per frame specifies the error in the frames while processing.

Figure 8, shows the correlation between the various restored video frames of the video sequence. The Time Vs correlation defines the change of the frame correlation with respect to the time of the various frames of the video sequence.

\section{Conclusion}

Video is basically the collection of the frames that are moved at much a fast speed that human eye perceives that it is actually moving. This paper discuss about the proposed method that is based on the DEFADE filter using the BPDM_CMF probability estimation technique. Performance of the proposed system is analyzed on the basis of the various parameters.

\section{References}

1. Lopez-Molina C, Baets BD, Bustinc H, Sanz J, Barrenechea E. Multiscale edge detection based on Gaussian smoothing and edge tracking. Knowledge-Based Systems, Elsevier. 2013 May; 44:101-11.

2. Jadav RA, Patel SS. Application of singular value decomposition in image processing. Indian Journal of Science and Technology. 2010 Feb; 3(2):148-50. DOI:10.17485/ ijst/2010/v3i2/29667.

3. Parthasarathi V, Surya M, Akshay B, Siva KM, Vasudevan SK. Smart control of traffic signal system using image processing. Indian Journal of Science and Technology. 2015 Jul; 8(16):1-5. DOI:10.17485/ijst/2015/v8i16/64622.

4. Vadhanam BRJ, Mohan S, Sugumaran V. Application of artificial immune recognition system for identification of advertisement video frames using BICC features. Indian Journal of Science and Technology. 2016 Apr; 9(14):1-12. DOI:10.17485/ijst/2016/v9i14/86635

5. Wang Y, Doherty JF, Dyck REV. Moving object tracking in video. In the Proceedings of 29th Applied Imagery Pattern Recognition Workshop, Institute of Electrical and Electronics Engineers (IEEE); 2000 Oct 16-18. p. 95-101.

6. Cai Z, Wen L, Lei Z, Vasconcelos N, Li SZ. Robust deformable and occluded object tracking with dynamic graph. Institute of Electrical and Electronics Engineers (IEEE) Transactions on Image Processing. 2014 Dec; 23(12):5497509. 
7. Yoon JH, Yang MH, Lim J, Yoon KJ. Bayesian multi-object tracking using motion context from multiple objects. In the Conference on Applications of Computer Vision, Institute of Electrical and Electronics Engineers (IEEE); 2015 Jan 5-9. p. 33-40.

8. Manikandan R, Ramakrishnan R. Human object detection and tracking using background subtraction for sports applications. International Journal of Advanced Research in Computer and Communication Engineering. 2013 Oct; 2(10):4077-80.

9. Malatesh SH, Gayathri V. Tracking moving objects on video analytics. International Journal of Research in Computer Engineering and Electronics. 2014 Nov-Dec; 3(6):1-5.

10. Luo W, Xing J, Zhang X, Zhao X, Kim TK. Multiple Object Tracking: A Literature Review. Computer Vision and Pattern Recognition [Internet]. 2014 [updated 2015 Sep 21; cited 2014 Sep 26]. Available from: https://arxiv.org/ abs/1409.7618

11. Wang Q, Fang J, Yuan Y. Multi-cue based tracking. Neurocomputing, Elsevier. 2014 May 5; 131:227-36.
12. Zhu S, Gu M, Liu J. Moving vehicle detection and tracking algorithm in traffic video. 2013 Jun; 11(6):3053-9.

13. Smeulders AWM, Chu DM, Dehghan A, Shah M. Visual tracking: an experimental survey. Institute of Electrical and Electronics Engineers (IEEE) Transactions on Pattern Analysis and Machine Intelligence. 2014 Jul; 36(7):144268.

14. Zhang S, Yao H, Sun X, Lu X. Sparse coding based visual tracking: Review and experimental comparison. Pattern Recognition, Elesvier. 2013 Jul; 46(7):1772-88.

15. Chen E, Haik O, Yitzhaky Y. Detecting and tracking moving objects in long-distance imaging through turbulent medium. Applied Optics. 2014; 53(6):1181-90.

16. Santosh DHH, Venkatesh P, Poornesh P, Rao LN, Kumar NA. Tracking multiple moving objects using gaussian mixture model. International Journal of Soft Computing and Engineering (IJSCE). 2013 May; 3(2):114-9. 